\title{
GONZALO ROBLES. UN TESTIGO DE LAS REVOLUCIONES AGRÍCOLAS GLOBALES
}

\author{
Marcela Dávalos \\ Juan José Dávalos López
}

Recibido: 29/10/2014 Aceptado: 05/05/2015

\begin{abstract}
Resumen
Gonzalo Robles nació en Costa Rica, pero la vida lo implantó en México. Fue testigo del declive de la dictadura porfirista y participó activamente en la construcción de las nuevas instituciones nacionales mexicanas -agrarias, industriales, financieras, educativas y culturales. Por su formación de ingeniero agrónomo y sus brillantes intervenciones durante la efervescencia revolucionaria, fue llamado por los presidentes Álvaro Obregón y Plutarco Elías Calles para crear escuelas y comunidades agrícolas. Y para ello, en un país devastado, fue enviado a recolectar las experiencias agrícolas en otros países. Sus registros de aquellos viajes dejan ver el complejo mapa del ámbito agrícola que Robles dibujó a su paso por dos continentes, tanto como muestran lo que era desplazarse por el mundo antes de la aviación. Asimismo, esos viajes, realizados entre 1923 y 1924, son prueba de una incipiente globalización. En un mundo todavía conformado por culturas locales, las ferias, exposiciones, ligas o sociedades internacionales fueron muestra, en aquel tiempo, de cierta homologación que cien años después conoceríamos como globalización. Aquí se enfatiza sobre todo una de sus propuestas: las vinculadas con el campo mexicano. No obstante, se señalan algunas de sus intervenciones en la industria, la banca o la cultura. Sus notas y estudios se conservan en el Fondo Gonzalo Robles del Archivo General de la Nación de México.
\end{abstract}

Palabras clave: Gonzalo Robles; Revolución mexicana; globalización; viajes; escuelas agrícolas; comunidades campesinas.

\begin{abstract}
Gonzalo Robles was born in Costa Rica but life took him to Mexico. He witnessed the decline of the Diaz dictatorship and participated very actively in the construction of the Mexican new national institutions -rural, industrial, financial, educative and cultural. By training in agronomy and brilliant interventions during the revolutionary ferment, he was called by the presidents Álvaro Obregón and Plutarco Elías Calles to create schools and farming communities. And for that, in a devastated country, he was sent to collect agricultural experiences in other countries. Their records of those trips reveal the complex map of agricultural field Robles drew on its way through two continents, as well as what was shown around the world before aviation. Also, these trips made between 1923 and 1924, are evidence of an incipient globalization. In a world still shaped by local cultures, Fairs, Exhibitions, Leagues and International Societies showed, at that time, a sample of what a hundred years
\end{abstract}




\begin{abstract}
later was called globalization. Here we emphasize one aspect of its proposals for the Mexican countryside, while globalizing policies; however we point out some of their activities in industry, banking or culture. His notes and studies are held in the Fund Gonzalo Robles General Archive of the Nation of Mexico.
\end{abstract}

Key word: Gonzalo Robles; Mexican Revolution; Globalization; trips; agricultural schools; rural communities.

En 1925, cuando Gonzalo Robles ocupó el puesto de director de Escuelas Centrales Agrícolas en México, habían pasado veinte años desde que dejó su tierra natal, Costa Rica. De Cartago salió rumbo a Chicago, con la idea de estudiar mientras vivía en casa de un pariente político al que su padre, Marcelino Robles Arias, había ayudado años atrás. Para agradecer aquel apoyo, Gonzalo debía permanecer en Chicago hasta acabar la carrera de ingeniero, sin embargo, en un acto de suerte impetuoso, se fugó de casa de su anfitrión sin previo aviso. ${ }^{1}$

Décadas después de su salida de Cartago y de haber sido un personaje público en la política mexicana, narró cómo se había desaparecido de Chicago para irse a vivir a Guadalajara con un tío paterno. Desde este momento, el azar lo fue implicando cada vez más en la creación de un país que estaba por hacerse y que permitía convertir en realidades las utopías revolucionarias de la generación a la cual perteneció. Ni la promesa de una exitosa carrera en Norteamérica, ni la civilización de acero lograron retenerlo; así, para 1909 ya estaba instalado en la ciudad de Guadalajara y había recorrido cientos de kilómetros.

En aquel tiempo, antes de que la aviación se convirtiera en un transporte común, trasladarse de país en país era una auténtica aventura. Esto es probablemente una explicación de sus diarios de viaje, en donde día tras día -y en ocasiones hora por hora-, Robles anotó su paso por cada puerto y país. Carreteras sinuosas, desfiladeros, veredas empantanadas, ríos sobre pangas o abismos cubiertos por neblina fueron algunos de los obstáculos que cruzó para llegar de la ciudad costarricense de Cartago al puerto caribeño de donde zarpaban los barcos de vapor que navegaban por el Atlántico. Embarcaciones que en las primeras décadas del siglo XX podían zarpar siempre y cuando las condiciones meteorológicas, las cuestiones técnicas y toda una gama de desavenencias permitieran cumplir con los horarios de la única línea que ofrecía dos viajes por semana a Nueva York y uno mensual a Veracruz.

Sin tener muy claro a dónde iba ir a estudiar, Gonzalo esperó en casa de su tío paterno, en la ciudad de Guadalajara, a cumplir dieciocho años, pues era un requisito para ingresar a la Escuela de Agricultura de San Jacinto, en un poblado muy cercano a la ciudad de México. Desde que salió de Costa Rica, en 1906, nunca más dejó de añadir kilómetros a sus recorridos, como tampoco dejó de tomar notas de lo que tuvo a la vista. En la escuela, ubicada en un viejo convento en el pueblo de Tacuba, se fue adhiriendo al proyecto de transformar la agricultura. Desde sus años formativos se identificó con los profesores que buscaban solución al acaparamiento de tierras, y desde entonces Gonzalo 
propuso que el sistema de propiedad en manos de grandes terratenientes pudiera fraccionarse y convertirse en comunidades campesinas autosuficientes. Así, al retomar el antiquísimo modelo de arrendamiento que se había empleado desde el siglo XVI en México, planteó la creación de pequeños productores agrícolas con la participación directa de los mismos campesinos. Sus notas muestran su pasión por registrar la diversidad de los campos y catalogar las distintas experiencias agrícolas, pero lo que en ese momento Robles aún no alcanzaba a ver era que la situación política que atravesaría México en las siguientes décadas lo atraparía, marcando con ello el resto de sus días.

La Escuela de Agricultura de San Jacinto, ubicada en lo que para entonces era un antiguo pueblo cercano a la ciudad de México, había sido creada desde el siglo XIX para educar a los "señoritos" que dirigían la economía de las grandes haciendas, no obstante, esa escuela fue particularmente sensible a las consignas de la Revolución mexicana. En 1910, el estudiantado en pleno firmó un documento en rechazo a la reelección del dictador Porfirio Díaz, en el cual se pedía su renuncia y se exigía la realización de elecciones. Del mismo modo, el grueso de los estudiantes apoyó la entrada triunfal del presidente Francisco I. Madero y también todos ellos fueron testigos de su asesinato. Luego de ese sangriento episodio, el estudiantado fue víctima de las políticas autoritarias del gobernante sucesor, Victoriano Huerta, un conservador impuesto mediante un golpe de Estado durante el periodo conocido como "La Decena Trágica". El apoyo político del plantel de San Jacinto a favor de la no reelección se revirtió en contra del estudiantado con medidas autoritarias que iban desde mantenerse durante días como guardias hasta la imposición de severos castigos, arrestos o aislamientos. Victoriano Huerta condenó al plantel a una vigilancia incesante que alteró las rutinas diarias de los internos. Molestos e inconformes por la militarización, por el corte presupuestal y por las sanciones autoritarias impuestas desde la presidencia a partir de 1912, los alumnos de la Escuela de Agricultura radicalizaron aún más sus criterios políticos.

A la rebeldía colectiva, que rechazó el castigo impuesto como venganza en contra del estudiantado por haber apoyado a Madero, a Gonzalo Robles se le sumó la rabia de saber que su único hermano, Enrique Robles, había muerto. Por su correspondencia se sabe que tenía apenas unos meses de haberse titulado en la Universidad de Baton Rouge, en Luisiana y de haber ganado un cargo en el departamento de medidores de la Stone Webster Engineering Corporation. Al enojo en contra de los "servicios de guardia obligatorios y vigilancia" o de estar entre las listas de "arrestados" (Gómez, 1976: 176-177), se añadía la indignación de que su único hermano hubiese muerto en medio de una confusión que no descartaba el asesinato.

Todo eso lo orilló a apuntalar sus posturas políticas. Luego de que de la Escuela de Agricultura se quedó sin fondos y debió sostener a los internos con las cosechas de los campos sembrados por ellos mismos, así como a alimentarse con las aves y el ganado antes destinados para la enseñanza, varios alumnos decidieron sumarse a las filas de la Revolución. Poco antes de que Victoriano Huerta asfixiara la escuela, varios estudiantes, entre ellos Robles, la dejaron para enlistarse en los contingentes que se dirigieron a Veracruz, para seguir al segundo nuevo líder revolucionario, Venustiano Carranza. 
La correspondencia entre Gonzalo y Enrique da pistas para reconstruir la frustración de no haber realizado los planes que hicieron de encontrarse en Estados Unidos, Costa Rica, América del Sur o México. Durante los siguientes meses Gonzalo caviló sobre las versiones de esa muerte, sucedida en un contexto de conflicto entre unos hombres de la mafia y la empresa Marconi (estación de radio fundada a finales del siglo XIX que fue cerrada por el Gobierno de EE. UU. en la Primera Guerra Mundial por cuestiones de seguridad). Enrique Robles había investigado cómo extender por mil la distancia de transmisión de sus ondas de radio. Su tesis, titulada Marconi Wireless Station for One Thousand Miles Range, había casualmente desaparecido, mientras que una mujer, de la que Enrique nunca habló, aparecía en los diarios como el móvil de su muerte. Con la escuela en quiebra y su hermano muerto, se dejó llevar en el movimiento revolucionario.

Gonzalo se fue a la Revolución sin saber la verdadera razón de aquella pérdida, pero también se fue sin saber que desde ese momento no pararía de viajar por el mundo. Los documentos que refieren a su paso por el ejército carrancista son casi inexistentes, no obstante, por contemporáneos suyos que escribieron al respecto, se conoce que la brigada en la que participó estaba comandada por el general Norzagaray, quien luego de estar en "el cuerpo del Ejército del Noroeste, se separó de Lucio Blanco en diciembre de 1914" (Cfr. Ulloa, 1986: 63). Robles llegó a esa tropa a través de Juan de Dios Bojórquez, un compañero de la Escuela de Agricultura "que se enroló con Carranza" ${ }^{2}$ y a quien Bojórquez había pedido que "llevara [a] algunos compañeros de la escuela para incorporarlos a su Estado Mayor... Le presenté a los ingenieros, Enrique Nájera y a Gonzalo Robles, a quienes les dio el grado de subtenientes"33 De modo que "se fueron a Veracruz un buen grupo de nuestros compañeros, entre los que puedo citarle a Enrique Nájera, Gregorio Díaz, Eustasio L. Contreras, Gonzalo Robles, Luis Arturo Romo, etcétera" (Cfr. Gómez, 1978: 453). En Veracruz, con menos de veinte años, Robles se topó por primera vez con los generales Álvaro Obregón (quien lo designaría pocos años después como director general de Agricultura), Salvador Alvarado, Heriberto Jara y Agustín Millán, así como a los humanistas Luis Cabrera, Jesús Urueta o Luis Manuel Rojas. Todos esos personajes, que participaron de distintas maneras en el movimiento revolucionario, estaban reunidos en un festejo provinciano de corte tradicional. Gonzalo fue uno de los que vieron cómo el presidente y su cortejo fueron recibidos con una "lluvia de flores y confeti arrojados desde los balcones y azoteas" (Cfr. Pasquel, 1972: 190).

Por narraciones de otros reclutas de la brigada Antúnez, se sabe que durante las interminables horas en las que el batallón se mantenía a la espera de nuevas órdenes militares, Gonzalo Robles tomaba el papel de cronista y relator. Por su parte, el general Norzagaray "invitaba con frecuencia al joven ayudante para que le expusiera la forma en que se desarrollaron los grandes hechos de la historia..." (Cfr. Emilio Alanis Patino, Gonzalo Robles, In Memorian: s.p.i.). Robles era un apasionado de las revoluciones inglesa y francesa, así como de los movimientos de emancipación, la literatura y el teatro. Esta fue la causa por la que el general decidió que "a este muchacho no lo llevaré a la campaña; lo dejaré en la capital, en el desempeño de otras comisiones... 
Aunque se ofenda lo dejaré aquí. Sería una lástima que se perdiera un hombre de esta categoría intelectual".4 (Cfr. Emilio Alanis Patino, Gonzalo Robles, In Memorian: s.p.i.).

Y, efectivamente, al terminar el cerco, Robles no prosiguió en la Avanzada del Norte en donde muchos de ellos murieron. Norzagaray decidió comisionarlo hacia los proyectos educativos. Y así Robles quedó fuera de los campos de batalla, en medio de un país arrasado. Los campos desiertos, las vías ferroviarias destrozadas, las ciudades arruinadas, la industria desmembrada y el comercio disminuido a trueque y a escalas locales.

Aun así, a pesar de ese escenario de muerte, una de las salidas tomadas por el gabinete del presidente constitucionalista Venustiano Carranza, fue partir desde cero y levantar al país desde experiencias revolucionarias. El presupuesto de los dos siguientes gobiernos fue retomar las prácticas agrarias previas de otros países y reconstruir desde cero la economía mexicana que, en esos años, era prioritariamente agrícola. Las enormes haciendas algodoneras, ganaderas, forrajeras, azucareras o cafetaleras, que antes de la Revolución estuvieron en manos de grandes terratenientes, hacendados y extranjeros, se vieron afectadas. Desde los propietarios despojados hasta los campesinos que no pocas veces se mantenían con pagos en especie, giraron sus parámetros durante aquel periodo revolucionario. Las estructuras agrarias anteriores perdieron sentido; por lo que se trató de estructurar todo desde el principio y en otra dirección. En otras palabras, se trataba de practicar uno de los lemas de los que la Revolución se había apropiado: "la tierra es de quien la trabaja", y para ello se destinaron actores en diversas direcciones.

Así, el Gobierno interino carrancista, establecido en el puerto de Veracruz, envió a sus cuadros a recorrer países con experiencias similares, a fin de enriquecer las leyes agrarias que Carranza decretó en 1915. En el momento en que Gonzalo Robles fue encomendado a recabar asuntos agrarios, se hallaba trabajando "como segundo ingeniero de la Comisión Local Agraria en el Estado de Veracruz” (Cfr. Economía..., 1982: 19). En junio el Gobierno Constitucionalista del Estado de Veracruz lo comisionó, junto con otras personas, "para que estudiara el funcionamiento de las escuelas de agricultura establecidas en Estados Unidos de Norte América”. (Cfr. Economía..., 1982: 20).

El universo de principios del siglo XX, apenas comunicado, en el que le tocó viajar, quedó grabado en sus cuadernos; en donde asentó tal cantidad de información que se alcanza a ver la diversidad y especificidades de las regiones y micro localidades agrícolas de aquel periodo. Ese primer viaje comenzó en las escuelas de agricultura de Estados Unidos y continuó con la escuela de Agricultura Práctica de Buenos Aires (Cfr. Economía..., 1982), que en ese momento era bien reconocida entre los agrónomos de avanzada por haber revolucionado sus sistemas de producción a partir del presupuesto de fortalecer el mundo agrario con el fin de "romper la aglomeración en los grandes centros" y "fijar a la población encariñándola con los trabajos del campo, de modo de detener el éxodo a las ciudades" (Cfr. Gutiérrez, 2007: 83). Después de los gobiernos personalistas y autoritarios, los fraudes electorales, las huelgas de agricultores y primeras protestas de los chacareros contra los terratenientes, las reformas electorales del presidente Roque Sáenz Peña y los intentos generales de revolucionar el sistema 
de propiedad agraria, los argentinos avanzaron en la transformación de la política y programas agrícolas tradicionales (Cfr. Hora Roy, 2002). Robles tomó notas de aquella efervescencia liberal y de las primeras elecciones libres en Argentina, además de cúmulos de información con la que hoy día sería posible trazar un mapa de la circulación de productos y mercancías en el mercado bonaerense de la primera década del siglo XX.

Los agrónomos de esa generación estaban convencidos de que el futuro del mundo se encerraba en el campo. Enlazados con el temperamento romántico propio de la época, que veía en la naturaleza y en las estructuras agrarias al germen de la felicidad y del progreso como el mejor sitio para educar a los hombres, concentraron todas sus fuerzas en alcanzar esa utopía. Con Zolá y Tolstoi bajo el brazo, siguieron esos sueños. Y Robles fue uno de ellos. Se interesó principalmente en reconocer y describir los métodos agrícolas revolucionarios, y con esa filosofía abarcaban todo. Al encumbrar a la tierra, a los campesinos y a la naturaleza, aquellos humanistas realzaban la importancia del ámbito agrícola, previo al fortalecimiento de la industrialización y del boom de las ciudades. Por eso, Gonzalo incluyó en sus notas cuanto vio a su paso. Para él no era posible referirse al mundo agrícola argentino sin registrar desde el Mercado Central de Frutos Avellaneda hasta la Universidad Popular de Boca, pasando por la Escuela de Granjeros, las pacas de lana, los conciertos o los edificios art decó. ${ }^{5}$

Aquel primer viaje fue una verdadera expedición. Durante las tres primeras décadas del siglo $\mathrm{XX}$, en un entorno mediado tan solo por el telégrafo, el FFCC, el barco de vapor y las largas epístolas, la conexión entre países requería forzosamente de la presencia de delegados, y Gonzalo Robles fungió como representante de cuanto tuviera relación con el universo de las técnicas agrícolas modernas. No obstante, a su regreso de Argentina, el Gobierno constitucionalista de Venustiano Carranza había ganado y se había trasladado a la ciudad de México. Las tareas y compromisos del nuevo Gobierno lo dejaron perplejo ante la indiferencia hacia sus investigaciones. Los directivos que le habían solicitado elaborar proyectos para México, al considerar las innovaciones agrícolas de otros países, se fueron y las instituciones quedaron disueltas; por lo que sus informes perdieron sentido. Fue entonces cuando Robles decidió guardar sus notas en el cajón, dejar la Comisión Local Agraria de Veracruz e irse a estudiar ingeniería civil a Indiana, en Estados Unidos.

En 1918, estando en la universidad de Valparaíso, recibió la noticia de que su padre, J. Marcelino Robles Arias, falleció. En ese momento el tiempo le cayó encima. Se percató de que llevaba más de diez años de no regresar a Costa Rica y de que ese 26 de julio había desaparecido el rostro de un anciano que probablemente no le hubiera reconocido; pues los escasos dieciséis años compartidos con él, apenas le alcanzaban para reconstruir algunos diálogos o pasajes de sus vidas compartidas. El dilema de regresar o no a Costa Rica fue imponente, tal como lo muestra la correspondencia con sus familiares y conocidos de Cartago. Luego de cartas, reflexiones, cuestionamientos o chantajes que lo invitaban a volver, la posibilidad de dejar México se disolvió nuevamente al verse envuelto en las vicisitudes de la Revolución mexicana.

Para 1920 se había titulado en la Universidad de Indiana, había regresado a la ciudad de México y se había reencontrado con algunos compañeros de la Escuela de 
Agricultura, quienes, al igual que él, serían parte de la joven generación que tejió las redes para reconstruir la economía nacional mexicana. Jesús Silva Herzog, Narciso Bassols, Daniel Cosío Villegas, Marte R. Gómez, Juan Ballesteros, Manuel Mesa Andraca, Miguel Yépez Solórzano, Juan de Dios Bojórquez, Gilberto Fábila, Ignacio L. Figueroa, Juan Ballesteros, Mario Bandala, Manuel Vázquez Díaz, Carlos Manuel Cox, Moisés I. de la Peña, Enrique Sarro, Pascual Gutiérrez Roldán, Ramón Fernández y Fernández, Federico Bach y muchísimos otros creían firmemente que su trabajo lograría reconstruir el espacio público. Todos ellos participaron en ese formato de circulación del saber global, formado por congresos, ferias, ligas, comités, encuentros y cuantas asociaciones colectivas internacionales ayudaran, en ese periodo, al fortalecimiento de la industria, las revoluciones y los procesos de cambio.

En ese ambiente, Robles se dio a conocer por sus participaciones en los Congresos Agronómicos realizados en la ciudad de México en septiembre de 1921 y de 1922. En esos eventos, inaugurados y apoyados por el ilustrado secretario de Educación, José Vasconcelos, se vertieron decenas de propuestas. Las de Gonzalo resultaron relevantes en uno de los temas álgidos de la Revolución: la subdivisión en pequeñas propiedades de los grandes latifundios que se habían fortalecido durante la segunda mitad del siglo XIX. En un clima en el que la gran mayoría de la población estaba implicada con el tema, sus propuestas tocaron emocionalmente al auditorio. Su ponencia, "Ideas generales para cooperar a la subdivisión del latifundio por medios indirectos", sostuvo que el latifundio se dividiera sin violencia y se integrara a la economía nacional, creando pequeños campesinos productores y propietarios que llevaran sus frutos al circuito mercantil, y así evitar intermediarios y acaparadores. Además, proponía crear "una propiedad menor", pero por "medios indirectos", es decir, de una manera menos drástica que la "directa". Con esto se aprovechaba la figura del "arrendamiento" -que existía en México desde el periodo colonial-; al mismo tiempo, Robles propuso crear una "obligación contractual" y un derecho para quienes no tenían tierra, en otras palabras, al responsabilizarse de un terruño, los campesinos se sentirían obligados a cuidarlo. En ese proceso se gestarían pequeños productores rurales, tal como él lo había registrado directamente y se realizaba en otros países. ${ }^{6}$

Luego del primer Congreso, Robles ya estaba inmerso en varios proyectos con colegas y amigos de la Escuela de Agricultura. Pocos años después, todos ellos ocuparían puestos clave. Uno de sus primeros proyectos fue crear un colectivo para difundir sus propuestas para el campo. Con Marte R. Gómez, Miguel Yépez Solórzano, Juan de Dios Bojórquez, Gilberto Fábila, Ignacio L. Figueroa, Juan Ballesteros y Mario Bandala redactaron un "programa de acción" y decidieron constituirse como Liga, crear un "Manifiesto" y un boletín al que bautizaron Germinal. Órgano de la Sociedad Agronómica Nacional. Curiosamente, entre 1910 y 1912, en San José de Costa Rica se había publicado una revista que también se titulaba Germinal (Ars, Veritas et Labor), la cual contenía además artículos literarios, de arte y de cultura general. No se sabe si hubo alguna influencia de aquel título en el Boletín, pero sin duda en ella se homenajeaba a Emile Zolá. ${ }^{7}$

$\mathrm{Su}$ propuesta era mejorar los productos del campo, el nivel de vida de los campesinos y artesanos, así como fortalecer cualquier acción que renovara "los focos 
dañados por la parálisis de las ideas, por el embotamiento de los sentimientos o por la prostitución de las virtudes" ("Editorial”, Germinal: 1922). ${ }^{8}$ En Germinal se publicaron artículos sobre el aprovechamiento de las aguas subterráneas del sur del Valle de México al lado de crónicas de Anatole France, pasajes de Napoleón Bonaparte, referencias a Tolstoi o a las artes agrícolas en el lejano Oriente. Para ellos, la técnica era un recurso que debía distribuirse universalmente, por lo que se convocó a expertos de cualquier parte del mundo, adheridos del "lado de los trabajadores" y "en contra de las oligarquías", a colaborar con ellos.

Su idea, siempre en busca de un vínculo con lo universal, fue apoyada por el presidente pro agrarista, Álvaro Obregón. Todas las condiciones estaban abiertas para facilitar la unión al movimiento mundial, tal como lo hicieron con un grupo de intelectuales franceses que buscaba "un mayor acercamiento con los trabajadores manuales". Ese colectivo había iniciado en la Casa de Cooperación de Lille y editaba un periódico semanal, dirigido por Henri Dumay, Le Progrès Civique. Journal de Critique Politique et perfectionnement social, que circulaba más allá de las fronteras de Francia. De inmediato se sintieron atraídos por su editorial. Las posturas radicales de ese diario que encabezaba cada una de sus páginas con el lema de "Journal honnête pour les honnêtes gens" (periódico honesto para gente honesta), cuadró con el ímpetu de Germinal, en un ambiente de crisis y vacío dejados por la Revolución.

Le Progrès y Germinal revelan las aspiraciones universalistas de aquella generación que parecía emplear modelos de comprensión transferibles para ambos lados del Atlántico. “No traficar ni con los poderosos ni con los humildes, ni con el oro de la tierra ni con las promesas de una vida venidera", ${ }^{9}$ así como adherirse al servicio del interés general, llamar "al mejoramiento y progreso de la agricultura nacional" o "influir cerca de los poderes públicos, como consejeros o como iniciadores, para que el fomento de la agricultura...sea de acuerdo con las conveniencias sociales e intereses de la Nación" ("Editorial", Germinal: 1922), ${ }^{10}$ eran proclamas compartidas entre ambas revistas.

En 1921, Gonzalo Robles fue nombrado director general de Agricultura por el recién presidente Álvaro Obregón. En su escritorio retomó de nuevo las investigaciones sobre Argentina y Estados Unidos que había dejado en el cajón en 1915, y estaba dispuesto a transformar las condiciones agrícolas de cada rincón de México. Así, entre noviembre de 1921 y mayo de 1922 tenía un cúmulo de correspondencia, folletos y bibliografía que había solicitado a cuantos ingenieros e instituciones agrícolas tuvo en mente: el registro de esos folletos, escuelas, asociaciones o grupos organizados de particulares, entre otros, son otra veta de documentación pendiente a investigar. En sus mensajes de solicitud había una concepción muy clara del campo y recabó detalles y minucias: programas escolares, precios de equipos, tipos de fertilizantes, diagramas de fertilidad, centros de investigación y todo cuanto se le ocurrió que podría favorecer al campo.

En menos de un año, su escritorio estaba ocupado con correspondencia e información enviada por los profesores y directivos de la The Ohmio State University, Cornell University, Portland Cement Asociation de Chicago, The Pennsylvania State 
College, United States Department of Agriculture, la Webb Publishing Company, Agriculture and Mechanical College de Oklahoma, New York State College of Agriculture o el Department of Parks de New York. Ese bombardeo postal les dejó catálogos, cuestionarios, procedimientos, calendarizaciones y cualquier cantidad de información sobre las actividades presentes, pasadas y futuras de esas instituciones. ${ }^{11}$

El Gobierno obregonista, siguiendo la lógica de recaba las experiencias ya asimiladas en otros países, promovió intercambios a fin de enriquecer los procesos revolucionarios. Fue Álvaro Obregón quien designó a Gonzalo Robles y a Juan Ballesteros para realizar un viaje que hoy sería inverosímil. Dada la formación agrícola de ambos, debían investigar sobre las escuelas, cooperativas, asociaciones, comunidades, etcétera, etcétera, en Europa, Rusia y América Latina. En junio de 1923, Robles y Ballesteros partieron como representantes de México para registrar, conocer y evaluar todo lo relacionado al ámbito agrícola de otros países. Obregón los envió a "viajar verdaderamente hasta el fin del mundo" para recabar información sobre la enseñanza y las técnicas empleadas, con vistas a implantarlas en México (Krauze, 1977). Literalmente recorrieron de polo a polo, con el objetivo de mejorar las fórmulas para la agricultura nacional mexicana.

Y Robles se tomó la tarea muy en serio. Los cuadernos que escribió día tras día durante los diecisiete meses que duró el viaje, son una invitación a pensar en los inicios de la globalización moderna. En aquel tiempo eran tan pocos los viajeros, que sus notas, además de dejar huella sobre la manera en la que se percibió el mundo agrícola durante las primeras décadas del siglo XX, sugieren hacer una historia de cómo se viajaba antes de las travesías aéreas. Desde su salida en tren de la ciudad de México hacia Veracruz, sus ritmos de viaje marcan una manera de percibir el tiempo y el espacio que resulta diametralmente opuesto a las expectativas de cualquier viajero contemporáneo. En el puerto veracruzano debieron esperar algunos días, hasta tener la certeza de que su barco estaba listo para zarpar. Nada aseguraba puntualidad, ni certeza. El mundo, fragmentado, apenas se unía con los itinerarios trazados por actores como Robles y Balleteros.

En 1923, cuando abordaron el navío que los llevó a Cuba -de la línea más conocida entonces, llamada la Hamburguesa Americana-, ${ }^{12}$ no existían salas de espera y la tripulación saludaba de mano a cada uno de los viajeros que subían al barco. Aquella línea de vapores había sido creada en 1847 y para 1909 la línea seguía anunciándose en los diarios costarricenses. De antemano, cualquier travesía internacional implicaba aceptar que de la convivencia cercana, durante semanas, con gente desconocida podrían emerger grandes coincidencias y amistades. A Gonzalo Robles no le resultó ajeno cruzar el mar rumbo al Caribe, no solo porque su tierra natal, Costa Rica, tenía como fronteras dos océanos, sino porque esa parte del trayecto lo reconocía ya por su anterior recorrido a Chicago. Los tres días que duró el itinerario de Veracruz a La Habana por mar, eran solamente la punta de un enorme iceberg que tardarían en recorrer más de un año.

De Cuba averiguaron todo lo posible. Los cuadernos de Robles testifican su interés por investigar lo relacionado con las técnicas agrícolas, así como de sus escuelas 
y prácticas colectivas en los campos, ya que atribuía al ámbito rural ser el principio de una economía justa. En ese momento, en la isla ya existían animadversión y rechazo al dominio por parte de Estados Unidos, aunque ningún cubano tenía idea de lo que vendría después. Era 1923 y el presidente Alfredo Zayas precedía los gobiernos militarizados de Machado y Batista; por su parte, Julio Antonio Mella encabezaba el rechazo al nombramiento de un general norteamericano como rector de la universidad y la crisis económica se intentaba cubrir con escuelas y caminos. Dos años después detonaría el movimiento que, a la postre, llevó a la Revolución cubana.

En la Escuela de Ingenieros Agrónomos, ubicada en la vieja Quinta de los Capitanes Generales, Robles clasificó especies que ni por casualidad insinuaban la existencia de semillas desechables. Sus caminatas por casi seis días entre escuelas, plantíos, praderas y playas, parecen haber sido una manera de equilibrar el cuerpo, antes de que las mareas mecieran al Florida East Coast, el barco que los llevó a la siguiente parte del trayecto a Miami. Sobre Cuba, Robles dejó notas que describen la flora tropical o la zafra tan detalladamente, que pareciese haber dejado también registro de los aromas.

Prosiguieron durante más de trescientas cincuenta millas por tren a lo largo de la costa este de Estados Unidos hasta Jacksonville, donde los esperaba una pequeña embarcación rumbo a Washington, D.C. En este lugar fueron mucho más que mal recibidos, pues entre otras cosas les fue negado cualquier acceso a instalaciones o información agrícola, debido a la tirante relación que México tenía con el país vecino. Sin saberlo, los funcionarios norteamericanos tomaron venganza, en revancha a la negativa de México de liquidar a sus ciudadanos que habían sido afectados con la expropiación de tierras y propiedades. La Junta programada con el Departamento de Agricultura y la Planta de inspección, desinfección y cuarentena, que era la responsable de "prohibir la entrada de fruta mexicana y cubana" (AGN, Fondo Gonzalo Robles: le negó el paso para evitar "que pudieran ser vehículos de plagas". ${ }^{13} \mathrm{El}$ sabor de boca amargo que les dejó la capital de EE.UU. fue desechado en las siguientes estaciones experimentales, colegios de agricultura, departamentos de entomología y jardines botánicos de Chesapeake Beach, Arlington, New Brunswick N.J., Baltimore y New York.

El 24 de julio "salimos de Nueva York por el Lehigh Valley a las 6:10 pm y llegamos el 25 a las 8:00 am a las Cataratas del Niágara" (AGN, Fondo Gonzalo Robles, Caja 54, exp. 3). ${ }^{14}$ Luego de estar un mes y una semana en Estados Unidos tomaron el Grand Trunk Railroad, la línea de ferrocarril canadiense para llegar, después de catorce horas de viaje, al límite con Canadá. Dos días de estancia, y antes de salir a "Ottawa vía Toronto por el Grand Trunk RR a las 5:50", el 26 de julio de 1923, Gonzalo elaboró una minuciosa reseña del Colegio de Agricultura de Gueph. Montreal y Quebec le habían dejado en claro el funcionamiento de la Estación Agrícola Central y un montón de ideas sobre las "veintitantas estaciones o granjas experimentales" del último país en donde estuvieron, antes de cruzar el Atlántico. ${ }^{15}$

En el último punto, el 28 el julio, estaban al norte del continente sorprendidos ante la expectativa de abordar uno de los trasatlánticos de la línea Canadian Pacific, que era uno de los más renombrados, en aquella época en la que la gente reconocía a los barcos por su nombre y origen. Aquel enorme buque pesaba catorce mil toneladas, 
tenía cupo para más de mil pasajeros, medía ciento cuarenta metros y la altura de sus mástiles parecía perderse en el cielo. Se trataba de un gigante de vapor, de los más rápidos de su época, que había participado en la Primera Guerra Mundial.

Hasta ese momento los diarios de Gonzalo Robles ya eran considerables, sin embargo, él sabía que la siguiente parte del viaje, la de atravesar el continente hasta llegar a Rusia, país en el que había nacido uno de sus autores preferidos, León Tolstoi, ocuparía la mayor parte de su atención. Luego de una travesía que los dejó en Southampton, Hamburgo y Berlín, pasaron a Letonia y Lituania, zona de influencia del país que en ese momento era un enigma para cualquiera que se considerara revolucionario. Durante los cuatro días de viaje en el Express del Norte que los llevó de Berlín a Riga y luego a Moscú, Robles no dejó de escribir. Cuando llegaron a la ciudad del Kremlin, en agosto de 1923, el Soviet Supremo, los Comisarios del Pueblo y el Politburó aún estaban acotados; la Constitución de la República todavía no era aprobada y la idea de que cada región del país tuviese sus propios representantes era tolerada. Rusia había apenas expulsado a los zares y abría sus puertas por primera vez a los países extranjeros con experiencias revolucionarias similares: representantes de México, Turquía o Alemania conocerían los campos experimentales del socialismo. Y Gonzalo Robles y Manuel Ballesteros fueron la primera comisión mexicana que pisó aquella tierra leninista. ${ }^{16}$

En la décadas de los veinte, cuando las exposiciones internacionales eran la manera de figurar y socializar en las incipientes industrias del mundo, Robles y Ballesteros fueron los representantes de México en la Primera Exposición Agrícola e Industrial de Moscú, promovida por el Estado bolchevique. Se trataba de reunir en una feria lo poco de la producción artesanal, textil y agraria que quedaba en pie en aquel país, luego de la revolución de octubre. En sus notas, Robles escribió detalladamente lo que vio en cada uno de los pabellones que anunciaban el futuro de aquel país socialista. A Moscú llegaron seres que parecían proceder del siglo XII. El lejano Oriente mandó caravanas de hombres en turbantes y pieles morenas que cargaban consigo siglos de historia. Robles anotó a su paso cuanto pudo, dejando constancia de un momento histórico irrepetible. Una taracea de razas que sin asombro mostraban sus extrañas diferencias: los mongoles curtidos en las estepas se mostraban altivos ante los habitantes de la república de Kirguistán. Frente a estos emergían los tártaros de la república autónoma de Crimea (viñedos, manzanos, aceites, pescados), las cabezas cubiertas con mascadas sobre los rostros blancos de las menonitas alemanas del Volga, los gorros de las estepas de los guerreros cosacos, las colonias judías que más de cinco siglos atrás, huyendo de Occidente, se establecieron en la región ucrano-lituana o los White Russians, todos ofrecían tantos productos como los que algún día transitaron por la ruta de la seda. En ese momento, Robles asentó, entre otros puntos, que “...con la exposición el gobierno ruso" (AGN, Fondo Gonzalo Robles, Caja 54, exp. 3, 1923) tenía, entre una de sus finalidades "la unificación de Rusia, haciendo que se conozcan unos a otros los distintos habitantes de las distintas regiones del país con sus productos y sus costumbres" (AGN, Fondo Gonzalo Robles, Caja 54, exp. 3, 1923). ${ }^{17}$ 
Mientras Gonzalo Robles leía el texto oficial presentado por la Comisión Mexicana en la Exposición, un reflejo inconsciente le reveló la importancia que tenía para él visitar la tierra del autor de La Guerra y la Paz: "antes de llegar aquí conocíamos a Rusia por su literatura jugosa y humana, por sus grandes apóstoles y por sus artistas", y no podíamos "despedirnos de Moscú", sin antes "pagar nuestro tributo de cariño al gran Apóstol ruso". Robles citaba con familiaridad y cariño al autor: "yo siempre he sido enemigo de la no violencia y si había algún ruso que me simpatizara era el loco este de Tolstoi que no creía en la resistencia al mal". ${ }^{18}$ Robles era un militante de la no violencia; era uno de esos lectores que asociaban al escritor ruso con Gandhi. Por eso, en cuanto pudieron, se fueron en eregrinación a Yasnaya Poliana, a conocer el recinto de Tolstoi, a ese lugar de nombre que ya "nos era familiar de niños, y que, mal pronunciado en nuestra lengua, tiene suavidades de promesa". ${ }^{19}$

Lo que en ese momento Gonzalo no se esperaba era encontrarse una enorme grieta en el país al que consideraba modelo para cambiar al mundo. En 1923, cuando Robles conoció a la hija menor de León Tolstoi, tenía muy clara su admiración por ese enorme personaje y apóstol pacifista que, además de renunciar a la opulencia, construir una escuela en concordia con la naturaleza, apelar respeto a las labores campesinas o haber sido excomulgado por la Iglesia ortodoxa cristiana, escribió las novelas que Gonzalo había cultivado letra por letra y le habían llevado a una filosofía de vida ajena a la violencia, adversa a la ostentación y crítica a la Iglesia.

En ese año había pasado el clímax de la Revolución de 1917, el tiempo de persecución política apenas asomaba en el extranjero y aún no se vislumbraba la dictadura de Stalin. No obstante, alcanzaron a sentir las tensiones de una guerra civil, las críticas al Ejército Rojo y las acusaciones contra los bolcheviques que poco después se convertirían en acechos tecnológicos, espionajes policiacos y estrategias persecutorias masivas. Durante su estancia, los Talleres Artísticos y Técnicos Libres que clamaban por el arte como parte de la vida y creación del mundo, aún estaban vivos; la "doctrina de la no-violencia" de León Tolstoi había sido "restituida" para la revolución; el país entero, aunque devastado por la guerra, el hambre y la muerte, anunciaba su Nueva Política Económica (NEP).

Pero todo esto estaba amenazado. Las pluralidades culturales, las repúblicas indómitas, la reunión de las diversidades productivas y todo cuanto a Robles le había parecido un presagio para reunir la producción artesanal y agrícola como una esperanza mundial, se reveló agrietada. La Exposición Agrícola e Industrial de 1923 comenzó a desvanecerse. La tramoya montada para que cada una de las repúblicas rusas expusiera y ofreciera sus mercancías y trabajo, ignoraba lo que se estaba fraguando. Además desconocía la importancia de aquella escenografía irrepetible. Ninguno de los actores en ese momento imaginaba que las diversidades y contrastantes culturas podrían sucumbir. Nadie podía tener en mente que décadas después, con la globalización universal, los habitantes de las estepas podrían ser igualados a los de San Petersburgo, los de Turkemistán, los Kirguises o los tártaros de Crimea.

A la diversidad escenográfica registrada por Robles, se sumaron sus conversaciones con Natalia Sedova, la esposa de Trotsky, quien era la directora de Cultura 
en el Ministerio de Educación, con Anatoli Lunatcharsky, el crítico Comisario de Instrucción Pública quien, junto a Alexander Bogdanov, había sido uno de los principales fundadores del movimiento artístico proletario. Anatoli-el disidente bolchevique que antes de ocupar el cargo de educación tuvo una relación de discrepancias y acercamientos con Lenin- los acompañó en varias ocasiones. Ellos no estaban de acuerdo con que el Ejército Rojo se convirtiera en partido, así como con de acusaciones que les caía por coordinar campañas masivas de alfabetización, por luchar por el rescate de numerosos monumentos históricos que los bolcheviques querían destruir, así como de la existencia del grupo Proletkult -en el que orbitaron artistas como Vladimir Tatlin, Aleksandr Ródchenko, Naum Gabo o Vladimir Mayakovsky-, el cual clamaba por apartar el arte del mercado de consumo y convertirlo en un bien colectivo.

Los hombres y mujeres con los que Robles y Ballesteros convivieron durante los veintidós días en Rusia, fueron deportados poco después. A Gonzalo le tocó confrontar emociones con los que meses más tarde serían los disidentes del Gobierno stalinista. Casi todos los delegados con quienes trató durante ese mítico e inverosímil viaje, tuvieron que huir. Y a todo esto se añadió la conmoción que se llevó en Yasnaia Poliana, cuando la hija menor de Tolstoi, Alexandra Tolstaya (mejor conocida como Sasha) le detalló los padecimientos que se gestaban en la tierra del autor de Ana Karenina. Además, fue Sasha quien le habló de la persecución y encarcelamientos: ella misma había sido presa de los bolcheviques por manifestarse a favor de la libertad de expresión. Las conversaciones con ella le dejaron claro el clima de vigilancia o la persecución de campesinos de la que poco se hablaba en el extranjero. Así se enteró de cómo la mansión de Yasnaia y los cuartos de piedra de la escuela agrícola, que Tolstoi había construido con ayuda de los campesinos de la región, habían sido saqueados y nacionalizados, mientras Saha fue encarcelada. Luego de ser interrogada, señalada como anarquista y puesta en libertad bajo vigilancia, se le permitió ser la directora de unas paredes, ya convertidas en museo, que eran de la casa donde ella misma había crecido.

Meses después de su estancia en Moscú, el país de la Internacional Comunista Leninista y del poder de los Sóviets al pueblo, había terminado: Lenin habría muerto y varias de las personas con las que había charlado por horas eran perseguidos políticos. Varios artistas de vanguardia, como Chagall, Kandinsky o Gabo se habían exiliado forzosamente o suicidado, como Sérguéi Esenin, el poeta del "mesianismo campesino". Para 1929 Sasha había huido a EE. UU. y ayudado a varios intelectuales a escapar, como Nabokov o Rachmaninoff. En la URSS el arte se había convertido en un sistema persecutorio, resguardado tras el lema del movimiento artístico proletario. Las escuelas tolstoianas para campesinos fueron vistas como un mesianismo contrarrevolucionario y las campañas de Lunatcharsky en defensa de los monumentos históricos que los bolcheviques querían destruir habían quedado atrás.

Con la distancia y desengaño de la revolución rusa, sin perder las esperanzas de construir escuelas de enseñanza agrícola vinculadas a cooperativas y dirigidas directamente por los campesinos, Robles continuó su camino en busca de experiencias acumuladas en otros países. El 12 de septiembre, cuando partieron rumbo a Petrogrado, se quedó meditando la intensidad de aquel contacto con Sasha, la nieta de 
Tolstoi y con la escuela para campesinos, Yasnaia Poliana, en la que el mismo escritor convirtió en su casa y religión. Esa experiencia sería otra de las que lo dejarían marcado el resto de sus días.

El 25 de septiembre de 1923 estaban ya en Finlandia con los pasaportes listos para pasar en el vapor Aeolus, a Estocolmo. La pulsión escriturística de Robles continuaría sin cesar. El 13 de octubre estaba tomando nota de la historia y del funcionamiento de la Sociedad Cooperativa de Mayoreo en Copenhague, la cual se había fundado en 1896 y luego de fusionarse reunió a dos mil cooperativas de consumo que incluyen casi la mitad de los hogares daneses. La cooperativa que vendía sus productos entre sus mismos miembros, estaba organizada por un comité electo en asamblea general, beneficiaba a sus integrantes, ya que realizaban ventas a precio de mercado, repartían las ganancias entre los miembros proporcionalmente a sus compras, asumían responsabilidad conjunta ilimitada, etcétera. Al día siguiente, mientras conversaba con el director, Niels Frederiksen, Robles anotó que el cooperativismo no tiene miras socialistas en tanto no es posible desterrar de golpe al comerciante particular, pero el movimiento cooperativista progresa sin predominio del régimen de gran propiedad. Con esas preguntas y sin perder de vista los vínculos con México -la sociedad importa anualmente 16,000 sacos de café y 800 toneladas de henequén, está dispuesta a ponerse en contacto con productores mexicanos. ${ }^{20}$ Robles continuó sus notas en Upsala, Gotemburgo, Malmo, Askoy, Esbjerg, Londres, Rennes, Bruselas, La Haya, Utrech, Berlín, Wagen o Venecia donde sus notas sobre la Colonia Agrícola Virgilio dan cierto tono melancólico al referir que es "para los huérfanos y huérfanas de guerra", pero que había sido "originalmente fundado en 1884 para huérfanos de inundación". ${ }^{21}$ Robles y Ballesteros hicieron una breve pausa, durante la Navidad, en Ámsterdam. Todo el año siguiente, su taquigrafía pareció estar marcada por cierto estado de ánimo, pero sus notas pasaron por España y siguieron hasta Portugal, el último país del viejo continente en donde Robles una vez más anotó todo cuanto pudo sobre el Instituto Superior de Agronomía, la dirección general de Agricultura, la Estación Agrícola Central de Lisboa y cuanto estuviera vinculado a la posibilidad de conformar cooperativas agrícolas conducidas por los campesinos. Último punto de Europa en donde a las seis de la tarde abordaron el Polonio rumbo a Río de Janeiro. Notas que continuaron en América del Sur.

Para mayo de 1925, Robles estaba en Río de Janeiro analizando los tipos de bosques y zonas de labranza; los "patronatos agrícolas" que "tratan de resolver el problema de los niños abandonados" a través de la enseñanza de la siembra y la cosecha $^{22}$. La abundancia de registros continuaron en Buenos Aires, Mendoza, Santiago, Valparaíso, Iquique, Antofagasta o Montevideo, en cada hoja quedaron rastros de yucas, café, algodón, alfalfa, aguacate, mejillones, salitre, presas naturales, cascadas y un raudal de lugares, situaciones, personas, condiciones y reflexiones que se expandieron desde Punta del Fuego hasta la Bahía de Hudson. De La Habana a Finlandia y de Iquique a Letonia, sus notas, tomadas de junio de 1923 a noviembre de 1924, son el resguardo de una época que cifró gran parte de sus esperanzas en el campo y en las comunidades agrícolas. 
Robles fue parte de una generación que dialogó con las dos guerras mundiales, el ascenso de los fascismos, la proyección del socialismo, la democracia y las transformaciones tecnológicas. Su interés primordial por los temas agrarios y su experiencia lo fue llevando a ocupar puestos clave en diversos ámbitos de la economía y la sociedad, sin nunca dejar de interesarse por los pequeños microcosmos agrícolas que producían la riqueza. Cuando Gonzalo regresó de aquel viaje inverosímil, el presidente Obregón había terminado su cargo, y en su lugar se hallaba Plutarco Elías Calles, otro político que provenía y representaba al mundo agrícola mexicano.

Desde que en 1921 ocupó el puesto de director general de Agricultura hasta 1935, cuando fungió como director del Banco de México, nunca dejó de poner especial atención en que los ejidatarios contaran con las plantas industriales para beneficiar sus cosechas, así como para asegurar que los créditos fueran otorgados a "ejidatarios o pequeños propietarios que cultivaran sus pertenencias por administración personal y directa, sin el empleo de peones", a fin de que el capital llegara a los "verdaderos agricultores de tipo campesino" (Manuel Mesa Andraca, "Gonzalo Robles, ilustre agrónomo", Nota cronológica de periódico nacional, s. f.). ${ }^{23}$

En el norte de México aspiró a revivir la destreza de los indígenas que algún día emplearon las hierbas y zacates que crecían alrededor de los lagos. La lechuguilla y la palma de los estados de Nuevo León, Coahuila y Zacatecas se convirtieron en comuneros y dueños de ejidos a quienes visitó y entrevistó, siempre tomando muestras de los suelos y de la naturaleza que los rodeaba, así como de los productos que tradicionalmente comerciaban. Para él, la reconstrucción nacional comenzaba con la autosuficiencia de comunidades capaces de producir, con las materias primas regionales, productos que se intercambiaran con otras regiones, tal como el sureste de México, donde tradicionalmente se empleaban cactus, yucas, henequén o palmas para elaborar sombreros, sogas o bolsas. Lo mismo ocurría con el cacao, anís, jamaica, quina, hule, piña, naranja, caña, papaya, garbanzo, manzano, plátano, maíz, etcétera.

Robles convergió con los jóvenes de la generación de 1915 que nacieron “entre 1891 y 1905... y que entre el mundo militar y político de la Revolución sufrían el tener ocasión de deslizar un ideal" (Gómez Mont, 1977: 45). Asimismo, asistió en el diálogo contra los científicos del porfiriato y abonó a las raíces agrarias de la Revolución, durante el periodo que apenas asomó a los frentes militares a la etapa constructiva de instituciones (Arciniega, 1977). Todos esos jóvenes de la generación de 1915 fueron marcados por "haber contemplado la Revolución sin participar en ella...una oportunidad de ordenar, de actuar, de encauzar" (Krauze, 1981: 29-30). Ellos fueron los últimos que polemizaron en contra de la opinión de sus mayores sobre las ventajas o no de los gustos afrancesados que habían predominado en México por más de cuatro décadas; además, ellos ese oponían a la idea de que la solución del país estaba en los capitales ingleses, holandeses o alemanes; ellos fueron los primeros en interesarse en la historia de los Estados Unidos y en la de los países del Este.

Por ello, la atención de Gonzalo Robles estuvo puesta en reunir la educación campesina y el apoyo financiero rural, tal como lo hizo al rescatar una escuela primaria e internado que años atrás había inaugurado Francisco I. Madero; la escuela de 
La Bolsa. A ella se refirió en un artículo cuando afirmó que "la escuela nueva está en gestación, la escuela que ha de curar los males mayores que aquejan al país: el parasitismo, el hambre y la ignorancia." ${ }^{24}$ En medio de no pocos conflictos, desde la Dirección de Escuelas Agrícolas y luego como gerente de los Bancos Agrícola o Hipotecario, logró convertir diversas haciendas -La Huerta en el Estado de Michoacán, la de San Roque en Guanajuato o la de Mexe en el Estado de Hidalgo- en escuelas vinculadas a las comunidades campesinas adyacentes, donde se compartía desde el trabajo común hasta la distribución de las ganancias. Durante décadas se le vio recorriendo la República mexicana de norte a sur y de oriente a poniente, en busca de créditos o apoyos que fortalecieran los circuitos agrícolas, con los mercantiles y los industriales. Su objetivo más profundo era fortalecer los productos regionales; aspiraba a vincular los henequenes con la grana cochinilla, al igual que los tequilas con los sopladores de vidrio.

En 1928, cuando ocupó la dirección del recién creado Banco Nacional de Crédito Agrícola, la posibilidad de encausar financiamientos lo llevó a armar un verdadero rompecabezas de la nación agrícola. De México, como él decía, no dejó sin pisar un solo cerro o costa, y nunca dejó de sorprenderle la engañosa suposición de que este país era rico en tierras cultivables. Si se trataba de abonos y fertilizantes, sus notas contemplaban por igual a grandes firmas, como Guanos y Fertilizantes, que a pequeñas plantas, como "El arbolito". En esos años enlazó a las fincas henequeneras de Yucatán con las de Haití y Cuba. Su apego a lo regional anticipó lo ecológico: el bagazo -pulpa potencialmente capaz de producir una cera similar a la de Carnauba- debía reutilizarse, por lo que conectó a los tejedores de fibras con los productores de las máquinas desfibradoras. Por la familiaridad que Campeche, Yucatán y Quintana Roo tenían con los cactus, yucas, henequén o palmas, buscó reunirlos para que avivaran los antiguos talleres que habían hecho famosos a los sombreros, sogas o bolsas del sureste, en los mercados estadounidenses y europeos. En agosto de 1931 contrajo matrimonio con Melanea Moreno Solórzano, una prima de la esposa de Lázaro Cárdenas, quien sería posteriormente presidente, y decidió apartarse de los cargos, pero durante su larga luna de miel tampoco dejó de registrar las notas que lo harían ser reconocido como el gran soñador del universo agrícola.

Hacia 1933 aquella generación constructora de instituciones se veía a sí misma como heredera de la Revolución Mexicana. Hasta entonces todos ellos habían sido partícipes de una época que alcanzó a ver los últimos pasos del antiguo régimen y las primeras pisadas de la modernidad. Todos ellos experimentaron lo que había sido transportarse únicamente por tierra y mar, al tiempo que fueron pioneros en volar. Asimismo, vivieron en jornadas marcadas tanto por la luz de las velas, como por los horarios prolongados de la luz eléctrica. Inmersos en esa experiencia de modernización que afectaba a los diversos niveles sociales, Robles fue parte de un grupo de letrados preocupados por editar obras y títulos en español; así, junto con Eduardo Villaseñor, Emigdio Martínez Adame, Daniel Cosío Villegas, Manuel Gómez Morín, Adolfo Prieto y Gonzalo Robles, concretaron, en septiembre de 1934, ${ }^{25}$ el convenio de una editorial que se convertiría en la más grande de México, el Fondo de Cultura Económica. 
En 1935, año crucial para la vida política de México por haberse iniciado el rompimiento político entre el general y caudillo Plutarco Elías Calles con el general Lázaro Cárdenas del Río -a quien Calles había respaldado para llegar a la presidencia-, Robles fue director del Banco de México por solo un año. Algunos de los motivos que explican su permanencia en el puesto solamente por un año, fue su condición de extranjero -su origen costarricense-, su filiación callista -al igual que Narciso Bassols- o bien el interés de otros actores por ocupar el cargo (Romero Kolbeck, 1982). Sin embargo, una vez terminado el sexenio del presidente Cárdenas, fue reincorporado al Banco de México, en el primer centro de investigación industrial. En esta institución trabajó durante cuarenta años, puesto en el que fundó e impulsó infinidad de proyectos, trazados desde la motivación de proyectar su ímpetu nacionalista en planes que permitieran a México modernizarse y formar parte protagónica de la sociedad mundial que se condensaba aceleradamente, sobre todo al término de la Segunda Guerra Mundial.

En esa etapa destaca el proceso que provocó la creación de la "Ley de Industrias Nuevas y Necesarias", en el Departamento de Estudios Industriales del Banco de México; en Banobras y Nacional Financiera, su papel para impulsar proyectos industriales y de investigación tecnológica; su participación en congresos de la Asociación de Bancos de México, así como el impulso de vínculos que coadyuvaron en asentar a la economía mexicana como referente para el desarrollo en América Latina en ese importante periodo, los cuales se registran en su intercambio e intelectual y profesional con Raúl Prebisch, conocido fundador de la Comisión Económica Para América Latina (CEPAL) de las Naciones Unidas (Prebisch, 1981).

Esa generación con la que Gonzalo compartió la vida nunca dudó de que el futuro individual dependía de culminar una carrera universitaria o de que la educación del pueblo era una clave para la prosperidad colectiva. Todos ellos coincidieron en la necesidad de construir un acervo cultural internacional, así como en recurrir a la tecnología como medio para modernizar y vincular a las diversas sociedades del mundo.

\section{Notas}

1 Archivo Familiar Gonzalo Robles, entrevista a Gonzalo Robles, Grabación, s. f.

2 Entrevista a Gonzalo Robles, Archivo Familiar, s/a, p. 24.

3 AGN, Fondo Gonzalo Robles, Caja 54, exp. 67.

4 Archivo Familiar Gonzalo Robles, citado por Emilio Alanís Patiño, Gonzalo Robles, In Memorian. Manuscrito. s.p.i (documento sin referencias).

$5 \quad$ AGN, Fondo Gonzalo Robles, Caja 53, exp. 45, s. f.

6 AGN, Fondo Gonzalo Robles, Caja 53, exp. 56, septiembre de 1921.

7 Archivo Familiar Gonzalo Robles, "Editorial”, Germinal. Órgano de la Sociedad Agronómica Nacional, Revista Quincenal, 1ero, Febrero, 1922, Tomo I, Núm 2: 1. 
Por unos meses se creyó que Hortensia, la secretaria de Gonzalo Robles, tuvo una confusión en el nombre de la línea de vapores, debida a la traducción, sin embargo, la línea fue establecida en 1847. Cfr. Diario de Costa Rica, enero 22, 1885, p. 3. Todavía para 1909, la línea Hamburguesa Americana, con sus naves Sarnia, Sibiria, Prinz August Wiheim, y Prinz Joaquim, seguía en funcionamiento, anunciándose en los diarios costarricenses. Cfr. Actualidades, Semanario de Intereses Hispano-Americanos, San José de Costa Rica, Febrero 27, 1909, Año 1, N. 2.

AGN, Fondo Gonzalo Robles, Caja 54, exp. 3, p. 3.

AGN, Fondo Gonzalo Robles, Caja 54, exp. 3, p. 38.

AGN, Fondo Gonzalo Robles, Caja 54, exp. 3, 129.

AGN, Fondo Gonzalo Robles, Caja 54, exp. 3, 271.

AGN, Fondo Gonzalo Robles, Caja 54, exp. 3.

Archivo Familiar Gonzalo Robles, Manuel Mesa Andraca, "Gonzalo Robles, ilustre agrónomo", nota cronológica de periódico nacional, s. f.

Gonzalo Robles, ¡Hossana!", Germinal. Op. cit.: 13.

Víctor Díaz Arciniega, Historia de la Casa. Fondo de Cultura Económica (1934-1996), México, FCE, 1972: 52.

\section{Bibliografía}

Arciniega Díaz, Víctor. Querella por la cultura 'revolucionaria' (1925). México: Fondo de Cultura Económica, 2010.

Economía e industrialización: Ensayos y testimonios, homenaje a Gonzalo Robles. "Cronología". México: Fondo de Cultura Económica, 1982.

Germinal. Órgano de la Sociedad Agronómica Nacional, "Editorial", Revista Quincenal, Tomo I, Núm 2, 1922. 
Gómez Mont, María Teresa. Manuel Gómez Morín, 1915-1939: la raíz y la simiente de un proyecto nacional. México: Fondo de Cultura Económica, 1977.

Gómez R., Marte. Episodios de la vida de la Escuela Nacional de Agricultura. México: Colegio de Posgraduados de Chapingo, 1976. Cartas. Vida política contemporánea. México: Fondo de Cultura Económica, 1978.

Gutiérrez, Talía Violeta. Educación, Agro y Sociedad. Políticas educativas agrarias en la región Pampeana, 1897-1955. Bernal: Universidad Nacional de Quilmes, 2007.

Hora, Roy. Los terratenientes de la pampa argentina. Una historia social y política, 1860-1945. Buenos Aires: Siglo XXI, 2002.

Krauze, Enrique. "La escuela callista". Enrique Krauze (Jean Meyer y Cayetano Reyes, Colaboradores), Historia de la Revolución Mexicana. La reconstrucción económica. Periodo 1924-1928. México: El Colegio de México, 1977.

. "Cuatro estaciones de la cultura mexicana". Revista Vuelta, 5-60 (1981): 29-35.

Mesa Andraca, Manuel. "Gonzalo Robles, ilustre agrónomo", Nota cronológica de algún periódico nacional, s. f.)

Prebisch Raúl. "Reflexiones sobre la crisis del capitalismo y la inflación". Economía e industrialización: Ensayos y testimonios, homenaje a Gonzalo Robles. México: Fondo de Cultura Económica, 1982.

Romero Kolbeck, Gustavo. "Prólogo". Robles, Gonzalo. Ensayos sobre el desarrollo de México. México: Banco de México, S. A./Fondo de Cultura Económica, 1982.

Ulloa, Berta. Veracruz capital de la nación. 1914-1915. México: El Colegio de México/Gobierno del Estado de Veracruz.

Marcela Dávalos. Mexicana. Obtuvo su maestría en Historia en la Escuela Nacional de Antropología e Historia y su doctorado en Historia por la Universidad Iberoamericana. Desde el año de 1989 es investigadora en la Dirección de Estudios Históricos del Instituto Nacional de Antropología e Historia (INAH) y ha impartido cursos en universidades de la India, Brasil y Alemania, así como diversas ponencias y conferencias tanto nacionales como internacionales. Actualmente da clases en la Universidad Autónoma de la Ciudad de México.

Sus investigaciones abordan la historia del territorio, prácticas culturales y actores en la ciudad de México. Sus textos refieren a preguntas derivadas de la historiografía urbana, y van de los barrios al control del espacio, pasando por ciertos personajes claves que han participado en el proceso de construcción de la modernidad desde el siglo XVIII. Además fue coordinadora del suplemento BI-CENTENARIO, para la Revista Proceso, durante los años del 2010 y 2011. Coordinadora del Boletín Oficial del Instituto Nacional de Antropología e Historia, Orden, Policía y Militarización en las ciudades. Siglos XVI-XXI, México, INAH, enero-abril, 2012. Coordinadora del libro De márgenes, barrios y suburbios, México, INAH, 2010; entre otras publicaciones.

Contacto: mardavalo@gmail.com 
Juan José Dávalos López. Mexicano. Licenciado en Economía por la Facultad de Economía, con estudios de Maestría en Historia por la Facultad de Filosofía y Letras, ambos de la Universidad Autónoma de México. Es profesor en la Facultad de Economía de esa institución, en las Áreas de Economía Política e Historia Económica; así como en temas de Historia Social en la Universidad Obrera de México. Además es coordinador o autor de varias publicaciones, entre ellas: Reforma para el Saqueo. Foro Petróleo y Nación. México, Editorial Proceso, México, 2015. “Fortaleza y Debilidad de las Finanzas de Pemex", en PEMEX. Presente y Futuro, IIEc-UNAM /UAM/Unacar; México, 2009. "Las reservas petroleras de México: su valor para la Nación”, en Política Energética, colección "Agenda para el Desarrollo", volumen 8, Miguel Ángel Porrúa/ UNAM/LX Legislatura, México, 2006. "El sector energético en espera del cambio" en México en el primer año de gobierno de Vicente Fox, Universidad Autónoma de Zacatecas/ Miguel Ángel Porrúa, México, 2002 "La quiebra de ENRON: ¿Modelo para el siglo XXI?" en Revista Macroeconomía, enero, 2002 "La profecía del Chapopoltli. La energía en México en el siglo XX", Revista Proceso, suplemento Bi-Centenario, agosto 2010.

Entre sus cargos, ha sido Coordinador del Colegio de Profesores de la Facultad de Economía del 2004 al 2007; Secretario Académico de la Academia de Economía Política, Facultad de Economía, UNAM de 2007 a 2010; Fundador y Coordinador del Seminario El $1^{\text {er }}$ Centenario de la Fundación de la UNAM, 2007-2011; actualmente es $2^{\circ}$ Secretario en el Colegio de Economistas Metropolitanos y del Valle de México e Integrante de la Comisión Estatutos de la Federación de Colegios de Economistas de la República Mexicana.

Contacto: juanjosedavaloslopez@yahoo.com.mx 